\title{
No-visible-scar cholecystectomy
}

\author{
Tadeusz M. Wróblewski ${ }^{1}$, Sebastian Piotrowicz' ${ }^{1}$, Marcin Kotulski1 ${ }^{1}$, Piotr Gierej', Bogna Ziarkiewicz-Wróblewska ${ }^{2}$ \\ 1Department of General, Transplant and Liver Surgery, Warsaw Medical University, Poland \\ 2Department of Pathology, Warsaw Medical University, Poland
}

Videosurgery and other miniinvasive techniques 2010; 5 (4): 158-160 DOI: 10.5114 /wiitm.2010.18830

\begin{abstract}
Introduction: Single incision laparoscopic surgery (SILS) is a laparoscopic method providing a good cosmetic effect, but requiring the application of special ports and instruments enabling the surgeon to perform the procedure.

We report three-ports cholecystectomy through umbilical and suprapubic incisions performed with typical laparoscopic instruments which calls no-visible-scar cholecystectomy (NVSC).

Material and methods: Twenty patients with symptomatic cholelithiasis were qualified for NVSC. Typical $\mathrm{CO}_{2}$ pneumoperitoneum was done after umbilical skin incision. Two ports of $5 \mathrm{~mm}$ were inserted in the maximum external edges of this incision. After cystic duct and cystic artery dissection the right one was exchanged for a port of $11 \mathrm{~mm}$. The second incision for the 11-mm trocar for the laparoscope was done in the suprapubic median line within the hair area.

Results: Cholecystectomies were performed without any conversion to classical laparoscopic cholecystectomy (LCH) or open surgery. They were not technically identical due to the gradual improvement in the access and manipulation of instruments. The time of the intervention ranged from 2 hours during the introduction of the new method to 50 min for the last procedures. No postoperative complications were observed and all patients were discharged not later than after conventional LCH.

Conclusions: NVSC is a three-port laparoscopic intervention performed with typical laparoscopic instruments. It is more convenient for the surgeon than single incision $\mathrm{LCH}$, because the placement of the optic in the suprapubic region gives more space for the instruments. It also provides a very good cosmetic effect of the intervention. The described procedure is easy to learn and in case of technical problems additional ports can be applied (as in typical LCH).
\end{abstract}

Key words: laparoscopic cholecystectomy, SILS, DILS

\section{Introduction}

One of the advantages of laparoscopic surgery is the much better cosmetic effect in comparison to open methods. So, further new modifications of typical laparoscopic techniques continue to be developed, including the concept of NOTES, to create novisible-scar surgery.

Single incision laparoscopic surgery (SILS) cholecystectomy is an example, but it requires the application of three special small ports or special multi-chan- nel and new bent instruments to facilitate the operation [1-6].

In this report we present three-port cholecystectomy through two incisions performed with trocars and instruments used in typical laparoscopic cholecystectomy $(\mathrm{LCH})$. Two ports were introduced in the umbilicus and a third one (for the camera) through an additional suprapubic $1.5 \mathrm{~cm}$ incision. This modification of SILS gives the surgeon more space in the umbilical region and makes this intervention safer for the patient and more comfortable for the surgeon. 


\section{Material and methods}

Twenty patients (14 women, 6 men, aged 18-74 years) with symptomatic cholelithiasis were qualified for NVSC. Patients suspected of having common bile duct (CBD) stones were excluded.

All patients underwent general endotracheal anaesthesia typical for $\mathrm{LCH}$. Patients were placed slightly on the left side $\left(20^{\circ}\right)$ in a $30^{\circ}$ reverse Trendelenburg position. The surgeon stood between the patient's legs and the assistant at the left side of the patient.

Trocars typical for classical LCH were used: $5 \mathrm{~mm}$ (Covidien, Norwalk, USA) and $11 \mathrm{~mm}$ (non-disposable Olympus, Hamburg, Germany). After U-shape 3-cm long umbilical skin incision, $15-\mathrm{mmHg}$ pneumoperitoneum was created with a Veress needle. The first 5-mm trocar was inserted inside the left edge of the umbilical incision. Through this trocar a 5-mm angled $30^{\circ}$ telescope was placed. Under visual control, a second 11-mm trocar (Covidien, Norwalk, USA) for the camera was inserted slightly to the right of the suprapubic median line within the hair area. Then, the camera from the umbilicus was transferred to this trocar, and to improve the visibility it was changed for a $30^{\circ}$ angled, $10-\mathrm{mm}$ telescope. The third, 5-mm trocar (Covidien, Norwalk, USA) was introduced inside the right edge of the umbilical incision, as far as possible from the $5-\mathrm{mm}$, first inserted trocar.

Standard 5-mm Endo Clinch and dissector (Covidien, Norwalk, USA) were used. At first, straight needle suture 3-0 Monocryl (Ethicon, J\&J) was inserted in the midline of the abdominal wall to hang up the round ligament of the liver. Then a second, similar suture was inserted from the anterior axillary line site, just below the right costal margin, and the fundus of the gallbladder was stitched, moved superolaterally and fixed to the abdominal wall just after being attached on the outside of the straight needle. A third suture was inserted in the same mode through the abdomen $5 \mathrm{~cm}$ below the second one and the neck of the gallbladder was punctured to help in opening of Calot's triangle. The Endo Clinch from the right trocar grasped the gallbladder to open Calot's triangle for dissection. After dissection of the cystic duct and artery the $5-\mathrm{mm}$ trocar in the left umbilical port was changed for an 11-mm, metallic, non-disposable trocar. The mentioned structures were then clipped using a typical 10-mm clip applier and finally cut with laparoscopic shears. The gallbladder was dissected by monopolar electrocautery and the fixations were released. Haemostasis of the gallbladder site was done progressively by electrocautery. Rinsing and suction of the abdominal cavity was done if necessary. Next the camera was translocated to the 11-mm umbilical trocar and the gallbladder was removed using a typical crocodile grasper through the lower suprapubic trocar site. Through this orifice a $16 \mathrm{Fr}$ catheter could be inserted and placed into the gallbladder site, if drainage was necessary. Pneumoperitoneum desufflation was followed by closure of the fascia in the umbilicus and in the suprapubic port site by 1-0 PDS (Ethicon, J\&J). The skin was closed with nonabsorbable separated sutures in the umbilicus and purse-string sutures in the suprapubic incision.

\section{Results}

The 20 performed no-visible-scar cholecystectomies (NVSC) did not require conversion to classical LCH or open cholecystectomy in any case. They were not technically identical due to the gradual improvement in the access and manipulation of instruments. The time of the intervention ranged from 2 hours during the introduction of the new method to $50 \mathrm{~min}$ for the last procedures. There was no need to apply a drain in most cases (7/20).

No postoperative complications were observed and all patients were discharged on the second day after the intervention, like after conventional LCH. A follow-up examination was performed within one week after the intervention. The skin sutures were removed and replaced with strips. After one month of observation there were no visible scars on the abdomen. The patients' status was found to be similar as after conventional LCH. The pathologist reported chronic cholecystitis in 12 patients, cholecystitis with associated cholesterolosis in 5, and exacerbation of the cholecystitis in 3 patients.

\section{Conclusions}

NVSC is a three-port laparoscopic operation performed with typical laparoscopic instruments. It is more convenient for the surgeon than SILS cholecystectomy, because the placement of the optic in the suprapubic region gives more space for the instruments. The transfer of the camera from the umbilical 
port site to the suprapubic hairy area provides much more space for the instruments in this region than described by authors for all single access techniques [7-10]. The surgeon's work is easier, while exposure of Calot's triangle and gallbladder during NVSC is not as ideal as during classical LCH, but much better than in SILS patients. That is why additional fixation of the gallbladder fundus by straight needle suture was applied, which considerably ameliorated the exposure of Calot's triangle.

NVSC provides a very good cosmetic effect with no visible skin scars The additional trocar placed in the lower suprapubic hairy area did not leave any significant trace on the skin. The cosmetic effect is more important for patients than the short stay in hospital and very quick return to normal activity and to the job. This has been raised recently in many publications and conferences [11].

The described procedure is easy to learn and in case of technical problems additional ports can be applied (as in typical LCH). The possibility of common application of bent laparoscopic instruments is essential to further development of this method. It will not only provide more operative space, but will also make Calot's triangle preparation safer in the sense of avoiding CBD damage.

\section{References}

1. Michalik M, Orłowski M, Frask A, et al. NOTES cholecystectomy the first experience in Poland. Videosurgery and other miniinvasive techniques 2009; 4: 42-5.

2. Hać S, Kobiela J, Proczko-Markuszewska M, et al. Transvaginal cholecystectomy - NOTES - initial experience. Videosurgery and other miniinvasive techniques 2008; 3: 139-41.

3. Tacchino R, Greco F, Matera D. Single-incision laparoscopic cholecystectomy: surgery without a visible scar. Surg Endosc 2009; 23: 896-9.

4. Hodgett SE, Hernandez JM, Morton CA, et al. Laparoendoscopic single site (LESS) cholecystectomy. J Gastrointest Surg 2009; 13: 188-92.

5. Kommu SS, Rané A. Devices for laparoendoscopic single-site surgery in urology. Expert Rev Med Devices 2009; 6: 95-103.

6. Chamberlain RS, Sakpal SV. A comprehensive review of singleincision laparoscopic surgery (SILS) and natural orifice transluminal endoscopic surgery (NOTES) techniques for cholecystectomy. J Gastrointest Surg 2009; 13: 1733-40.

7. Kurpiewski W, Pesta W, Kowalczyk M, et al. SILS cholecystectomy - our first experiences. Videosurgery and other miniinvasive techniques 2009; 4: 91-9.

8. Michalik M, Frask A, Trybull A, Lech P, Witzling M. Methods of treatment for gallbladder diseases. Videosurgery and other miniinvasive techniques 2009; 4: 121-5.
9. Langwieler TE, Nimmesgern T, Back M. Single-port access in laparoscopic cholecystectomy. Surg Endosc 2009; 23: 1138-41.

10. Elazary R, Khalaileh A, Zamir G, et al. Single-trocar cholecystectomy using a flexible endoscope and articulating laparoscopic instruments: a bridge to NOTES or the final form? Surg Endosc 2009; 23: 969-72.

11. Stefaniak T. Adamczyk K, Walerzak A, et al. Body image is an important augmentation to quality of life in evaluation of the results of laparoscopic versus classic surgery. Videosurgery and other miniinvasive techniques 2010; 5: 146-51. 